\title{
Bayesian inference on the galactic magnetic field toward the south galactic pole using UHECRs
}

\author{
Jihyun Kim* \\ Ulsan National Institute of Science and Technology \\ Department of Physics, Ulsan National Institute of Science and Technology, Ulsan 44919, Korea \\ E-mail: jihyunkimeunist.ac.kr
}

\section{Hang Bae Kim}

Hanyang University

Department of Physics and The Research Institute of Natural Science, Hanyang University, Seoul 04763, Korea

E-mail: hokimehanyang.ac.kr

\section{Dongsu Ryu}

Ulsan National Institute of Science and Technology

Department of Physics, Ulsan National Institute of Science and Technology, Ulsan 44919, Korea

E-mail: ryu@sirius.unist.ac.kr

\begin{abstract}
Owing to the Pierre Auger Observatory (PAO), we have an unprecedented amount of ultra-highenergy cosmic ray (UHECR) data. Using the data, we study the influence of the galactic magnetic field (GMF) on the trajectory of UHECRs with energy above $6 \times 10^{19} \mathrm{eV}$. The GMF is not uniform, and its configuration is still uncertain. Besides, most studies on the GMF have been about the disk field. In this study, we focus on the GMF toward the south galactic pole (SGP), which is thoroughly within the field of view of the PAO. We examine the effects of the GMF on the arrival direction of UHECRs by statistical tests of correlation with the large-scale structure of the universe. The deflection angle of UHECRs affected by the GMF is inferred through the Bayesian inference with Monte-Carlo simulations. We present the estimated strength of the GMF toward the SGP based on the deflection angle and discuss the implications of our results.
\end{abstract}

35th International Cosmic Ray Conference - ICRC2017

10-20 July, 2017

Bexco, Busan, Korea

${ }^{*}$ Speaker. 


\section{Introduction}

Magnetic fields are ubiquitous in the universe and play an important role in many astrophysical phenomena. Lots of researchers have investigated cosmic magnetic fields using many different methods such as indirect observations in optical, infrared, and radio wavelengths, or through magnetohydrodynamic simulations (See the review of [1]). However, the origin and nature of magnetic fields in the universe is one of the unanswered questions in the modern astrophysics.

The magnetic fields in our galaxy are called the galactic magnetic fields (GMF) and those in the large-scale structure (LSS) of the universe, which is outside of the Milky Way, are named the intergalactic magnetic fields (IGMF). Though we mentioned that magnetic fields in the universe are not fully understood yet, we have some knowledge of the structure and strength of the GMF and IGMF by the combined efforts of the observations and simulations. The configuration of GMFs is not uniform, and the strength of the GMF depends on its position in the celestial sphere. The strength of the GMF is considered to be about a few $\mu \mathrm{G}$ [2][3]. In the case of IGMFs, unfortunately, it is more complicated to understand compared to the case of GMFs. The strengths of the IGMF vary from $\mathrm{nG}$ to $\mu \mathrm{G}$ in the LSS [3], which consists of groups and clusters of galaxies, filaments, and voids. Both GMF and IGMF strengths have model dependence, but there are wide discrepancies, several orders of magnitude, from one model to the other one in the case of the IGMF [3][4][5].

Meanwhile, it is important to understand the magnetic field in the universe for studying ultrahigh-energy cosmic rays (UHECRs), charged particles coming from outer space to the earth and especially with energies above $10^{18} \mathrm{eV}$. The trajectories of these UHECRs from their sources to the earth are affected by the magnetic fields in the universe. The deflection angle $(\theta)$ is governed by the strength of a magnetic field $(B)$ and the energy $(E)$ and atomic number $(Z)$ of the primary particle of the UHECRs, $\theta \propto Z B / E$. Though it is not easy to constrain on all the terms that affect the trajectory of UHECRs, theoretically, it is possible to probe the magnetic fields in the universe with the UHECRs.

Since the discovery of UHECRs, many observations have been conducted to find out the answer to the mysteries of UHECRs for more than 50 years. The observations of UHECRs are aimed at solving the problems of the mass composition of a primary particle, the existence of suppression in energy spectrum and the origin of UHECRs. Owing to these efforts, we have obtained some answers to the physics of UHECRs. Recently, the Greisen-Zatsepin-Kuzmin (GZK) suppression in the energy spectrum of UHECRs has been confirmed [6][7][8]. In addition, the results of mass composition show that the primary particles of UHECRs would be protons or light nuclei or a mixed composition [9][10][11].

In this work, based on the clues that we have confirmed as mentioned above, we will make a step forward to open the era of UHECR astronomy by using the unprecedented amount of UHECR data recorded by Pierre Auger Observatory (PAO). We infer the strength of GMFs from its influence on the trajectory of UHECRs with energies above $6 \times 10^{19} \mathrm{eV}$. In this work, we focus on the GMF toward the south galactic pole (SGP) because this region is thoroughly within the field of view of the PAO and has been less studied compared to the disk field [12]. We examine the effects of the GMF on the arrival direction of UHECRs using statistical tests of correlation with the LSS of the universe. The deflection angle of UHECRs affected by the GMF is inferred through the Bayesian inference with Monte-Carlo simulations. 


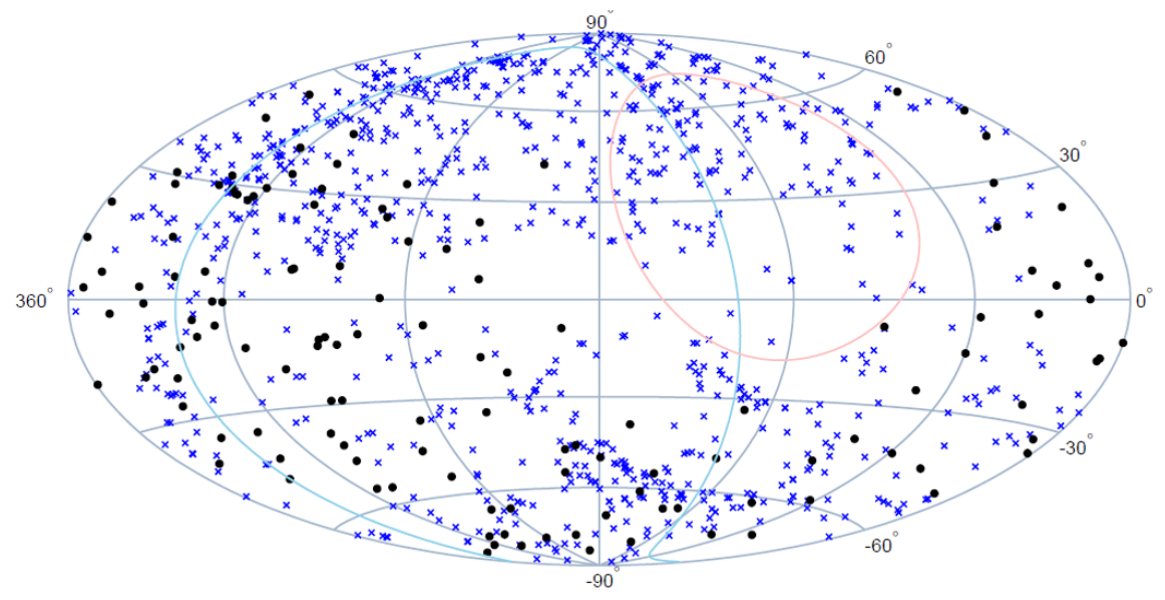

Figure 1: Hammer projection of skymap in the galactic coordinates. The blue $\mathrm{X}$ marks are the active galactic nuclei listed in the 13th edition of Veron-Cetty and Veron catalog [13]. The black dots are the ultra-highenergy cosmic rays that have energies above $6 \times 10^{19} \mathrm{eV}$ observed by Pierre Auger Observatory [14]. The pink and skyblue line represents the border of PAO's field of view and the supergalactic plane, respectively.

\section{Simulation model and Statistical test methods}

The sources of UHECRs are not confirmed yet, but they do belong to the LSS of the universe. Additionally, the maximum distance of the sources should be about $100 \mathrm{Mpc}$ because we use the UHECR events which have energies above $6 \times 10^{19} \mathrm{eV}$ which is higher than the GZK cutoff energy [15][16]. In this work, we assume that there is a correlation between the arrival direction distribution of UHECRs and the LSS of the universe. We generate mock UHECRs following the LSS of the universe and estimate the most plausible deflection angle by a statistical comparison of the correlation between the observed UHECRs and the LSS of the universe and that of the mock UHECR and the LSS of the universe.

\subsection{Data we used}

In this work, we pick the positions of active galactic nuclei (AGN) as the tracers of the LSS. The information on AGN is taken from the 13th edition of Veron-Cetty and Veron catalog [13], a compilation of all known AGN from various catalogs. We only use the AGN sample within the GZK radius, about $100 \mathrm{Mpc}$. The number of objects within this distance cut is 862 .

The UHECR data set we use is an up-to-date event observed by PAO published in 2015 [14]. In that paper, the $\mathrm{PAO}$ extends the zenith angle cut from $60^{\circ}$ to $80^{\circ}$; therefore, they have a field of view from $-90^{\circ}$ to $44.8^{\circ}$ in declination. The published data set includes the events which have energies above $5.2 \times 10^{19} \mathrm{eV}$, and the total number of data is 231 . This 10 -year data set gives us an unprecedented amount of UHECR data observed by a single observation project. Here, we only use the events that have energies above $6 \times 10^{19} \mathrm{eV}$, which is above the GZK cutoff energy. In Figure 1, the Hammer projection of skymap in the galactic coordinates is shown. We present the position of AGN within $100 \mathrm{Mpc}$ with the blue X marks and the UHECR events with energy $6 \times 10^{19} \mathrm{eV}$ with the black dots. 

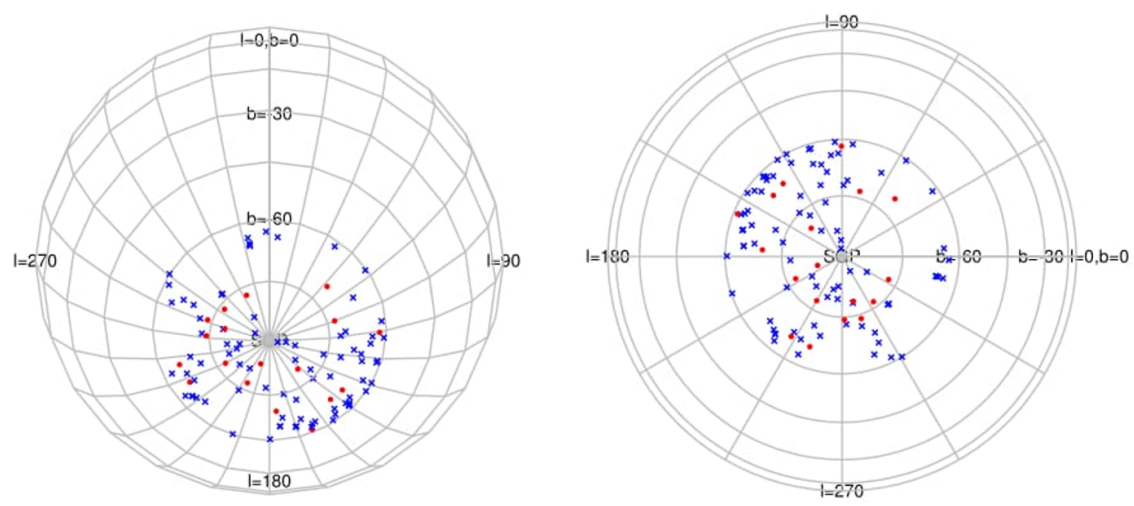

Figure 2: The distribution of $A G N$ and UHECR events which are observed in the region in which we are interested $\left(-90^{\circ} \leq b<-60^{\circ}\right)$. The blue $\mathrm{x}$ marks represent the AGNs, and the red dots indicate the UHECR events. The left panel is the galactic coordinates in the 3-dimensional plot, and the right panle is the orthographic projection centered on the south galactic pole.

Because the GMF is not uniform in the sky, it is not appropriate to estimate the strength of the GMF in all the sky at once by analyzing the effects of the GMF on the arrival direction distribution. Targeting a small area is a more efficacious and reasonable way to infer the strength of the GMF by using the arrival direction distribution of the UHECRs. Thus, we focus on the GMF toward the SGP, $-90^{\circ} \leq b<-60^{\circ}$, which is thoroughly within the field of view of the PAO. We only use the data toward the SGP. There are 83 AGNs and 18 UHECR events, which are shown in Figure 2. The blue $x$ marks represent the AGNs, and the red dots indicate the UHECR events. The 3-dimensional plot of the galactic coordinates are shown on the left, and on the right is the orthographic projection centered on the SGP.

\subsection{UHECR model}

To realize a set of mock UHECRs in the simulation, we assume two contributions come into the expected flux at a given arrival direction: a certain fraction of UHECRs with energy above $E_{c}$ originate from the source within a distance $d_{c}$, while the remaining fraction of them are from the isotropically distributed background contributions.

$$
F(\hat{\mathbf{r}})=F_{\mathrm{SRC}}(\hat{\mathbf{r}})+F_{\mathrm{ISO}},
$$

where $F_{\mathrm{SRC}}(\hat{\mathbf{r}})$ is the fraction originating from the source, and $F_{\mathrm{ISO}}$ is the isotropic component.

$$
f_{\mathrm{SRC}}(\hat{\mathbf{r}})=\sum_{i \in \mathrm{SRC}} \frac{L_{i}}{4 \pi d_{i}^{2}} \cdot \frac{\exp \left[-\left(\arccos \left(\hat{\mathbf{r}} \cdot \hat{\mathbf{r}}_{i}^{\prime}\right) / \theta_{s i}\right)^{2}\right]}{\pi \theta_{s i}^{2}},
$$

where $L_{i}$ is the UHECR luminosity, $d_{i}$ is the distance to the object, $\hat{\mathbf{r}}$ is the arrival direction of the UHECR, $\hat{\mathbf{r}}^{\prime}$ is the direction to the source object, and $\theta_{s}$ is the smearing angle from the source. We assume that all sources have the same UHECR luminosity, $L=L_{i}$, and the same smearing angle, $\theta_{s}=\theta_{s i}$. In the simulation, the smearing angle is taken to be a free parameter, from $0^{\circ}$ to $90^{\circ}$, 
and it will be interpreted as a deflection angle by the GMF. Here, we assume that the deflection by IGMF would not have a strong influence on the trajectories of UHECRs toward the region in which we are interested. We consider the deflection angle in the simulation following the Gaussian flux distribution with a certain angular width.

Also, we take into account the geometrical efficiency, a so-called exposure function. Due to the efficiency of the detector, there is a limit to the amount of the sky that can be observed by the detector. The field of view of the detector is determined by the location of the detector array and the zenith angle cut by the efficiency. Then the exposure function $h$ is a function of the declination $\delta$ only,

$$
h(\delta)=\frac{1}{\pi}\left[\sin \alpha_{m} \cos \lambda \cos \delta+\alpha_{m} \sin \lambda \sin \delta\right]
$$

where

$$
\alpha_{m}= \begin{cases}0, & \text { for } \xi>1, \\ \pi, & \text { for } \xi<-1, \text { with } \xi=\frac{\cos \theta_{m}-\sin \lambda \sin \delta}{\cos \lambda \cos \delta}, \\ \cos ^{-1} \xi, & \text { otherwise }\end{cases}
$$

$\lambda$ is the latitude of the detector array, and $\theta_{m}$ is the zenith angle cut due to the efficiency of the detector. The latitude of the PAO site is $\lambda=-35.20^{\circ}$, and the zenith angle cut of the data we used is $\theta_{m}=80^{\circ}$. In Figure 1, the pink line, the border of PAO's field of view, is determined by this exposure function.

\subsection{Comparison of arrival direction}

Considering the exposure function, we generate $10^{6}$ mock UHECR events based on the UHECR flux, $F(\hat{\mathbf{r}})$. Then, we calculate all the cross-correlational angular distance between the UHECR events and the LSS of the universe, represented by the AGNs. We call this quantity correlational angular distance distribution (CADD), which is written as [17][18],

$$
\left\{\cos \theta_{i j^{\prime}} \equiv \hat{\mathbf{r}}_{i} \cdot \hat{\mathbf{r}}_{j}^{\prime} \mid i=1, \ldots, N ; j=1, \ldots, M\right\},
$$

where $\hat{\mathbf{r}}_{i}$ is the arrival direction of the UHECR, which has a total of N events, and $\hat{\mathbf{r}}_{j}^{\prime}$ is the direction to the AGNs, which are M objects. Then, we obtain two sets of CADD from the observed UHECRs, $\mathrm{CADD}_{\mathrm{obs}}$, and from the mock UHECRs by the simulation, CADD $\mathrm{D}_{\text {exp. The }}$. TADDs show the feature of the correlational distribution. From the statistical comparison of these CADDs, we can test whether the model is likely to describe the distribution of observation or not. To compare two distributions, we employ the Kuiper statistic, $D_{\mathrm{KP}}$, which is the sum of the maximum difference of the observed distribution above and below the expected one,

$$
D_{\mathrm{KP}}=\max _{x}\left[P_{\mathrm{obs}}(x)-P_{\exp }(x)\right]+\max _{x}\left[P_{\exp }(x)-P_{\mathrm{obs}}(x)\right]
$$

where $P_{\text {obs }}(x)$ is the cumulative distribution of $\mathrm{CADD}_{\text {obs }}$ and $P_{\text {exp }}(x)$ is that of $\mathrm{CADD}_{\text {exp }}$. A small discrepancy in the two CADDs, $D_{\mathrm{KP}}$, shows that they have a similar distribution and they can be obtained from the same population. To get the probability that the observed UHECR data and the expected UHECR data are coming from a same population, we need to calculate the significance level of the Kuiper statistic. Through the Monte-Carlo simulations, we generate the same number of mock UHECRs as the observed data for the source model, which are reference sets. For each set 

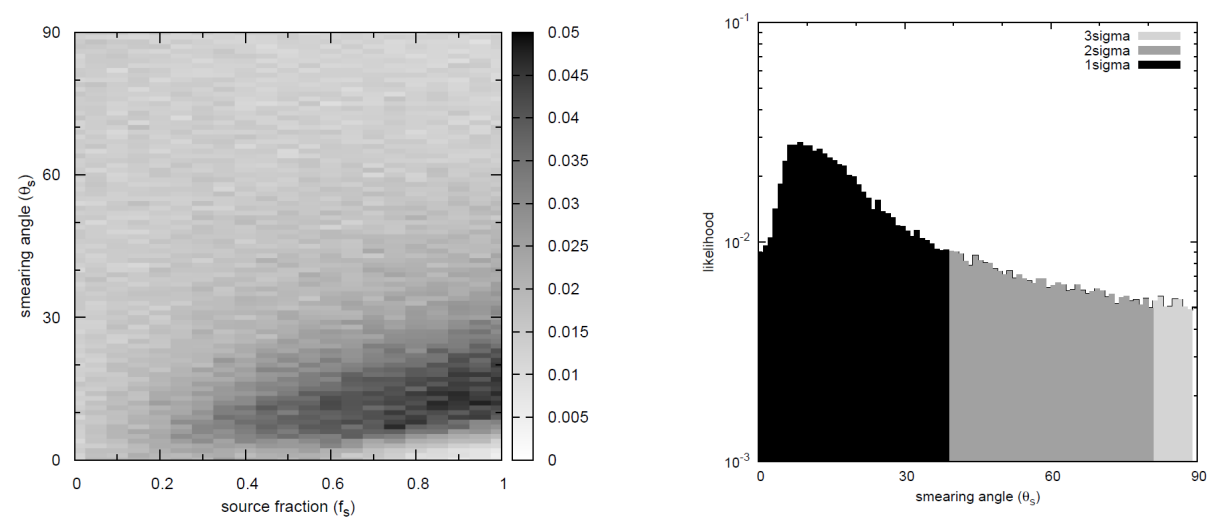

Figure 3: The left panel shows source fraction $\left(f_{s}\right)$ and smearing angle $\left(\theta_{s}\right)$ dependence of likelihood by the CADD method for the PAO data. The right panel is the credible interval of the smearing angle for the source fraction of 0.7 .

of mock UHECRs, we calculate the Kuiper statistic and repeat this procedure $10^{4}$ times. Now, we have $10^{4}$ sets of Kuiper statistics. Then, the significance of $D_{\text {obs }}$ can be inferred from the $10^{4} D_{\text {exp }}$ pool. Therefore, our probability estimate is reliable up to roughly $10^{-4}$.

\section{Analysis results}

In our simulation, we have two free parameters: the source fraction $f_{s}$ and the smearing angle $\theta_{s}$. The correlation is tested by the exploratory scan with $0 \leq f_{s} \leq 1$ and $0^{\circ} \leq \theta_{s} \leq 90^{\circ}$. The left panel of Figure 3 shows the likelihood dependence for the source fraction $f_{s}$ and the smearing angle $\theta_{s}$ by the CADD. The dark gray color represents the high likelihood of the given model. This result shows that the distribution of observed UHECR events toward the SGP is compatible with the source dominant model with the small smearing angle.

According to [19], for the UHECRs that have energies above $6 \times 10^{19} \mathrm{eV}$, about $30 \%$ of observed UHECRs are coming from the isotropic background beyond $100 \mathrm{Mpc}$. In other words, the source fraction is estimated to be 0.7 . From now on, we will take the fiducial value of $f_{s}=0.7$ to investigate the strength of the GMF toward the SGP.

The right panel of Figure 3 shows the credible interval of the smearing angle with the source fraction of 0.7. The color scale represents the sigma level of $1 \sigma, 2 \sigma$, and $3 \sigma$. For $1 \sigma$ level, the credible interval of the smearing angle is $0^{\circ} \leq \theta_{s} \leq 38^{\circ}$. The best-fit parameter is $8^{\circ}$. Based on this best-fit smearing angle, we will present the estimated strength of the GMF toward the SGP and will discuss the implications of our results in the next section.

\section{Discussion and Conclusion}

It is understood that the GMF consists of regular and turbulent components. Observationally, the strengths of turbulent components are stronger than those of regular one [1]. In this work, therefore, we estimate the strength of the GMF for the turbulent components, which gives an upper 
limit. The strength of the GMF can be estimated by

$$
B_{\mathrm{rms}}[\mu \mathrm{G}]=\frac{\theta_{\mathrm{tur}}}{0.83^{\circ}} \frac{1}{Z}\left(\frac{E}{6 \times 10^{19} \mathrm{eV}}\right)\left(\frac{10 \mathrm{kpc}}{D}\right)^{1 / 2}\left(\frac{100 \mathrm{pc}}{l_{c}}\right)^{1 / 2}
$$

where $\theta_{\text {tur }}$ is the deflection angle, $Z$ and $E$ are the atomic number and the energy of primary particle of UHECR respectively, $D$ is the size of the magnetic field extension, and $l_{c}$ is the average size of patches.

To infer the strength of the GMF, we need to set the atomic number of the primary particles. Determining the mass composition of UHECRs is one of the main objectives for observing UHECRs. Typically, it is inferred by using the maximum depth of an extensive air shower. According to the PAO [10], the analysis results of mass composition show that the primary particles of UHECRs are less likely to be heavy nuclei, like iron nuclei, rather they would be light nuclei or mixed compositions.

Adopting this result, if we assume the primary particles of UHECR are nitrogen nuclei $(\mathrm{Z}=7)$ which have energies $E=6 \times 10^{19} \mathrm{eV}$, we infer that the GMF strength toward the SGP would be $2.51 \mu \mathrm{G}$. Here, it is taken $D=3 \mathrm{kpc}$ and $l_{c}=100 \mathrm{pc}$ for the properties of the GMF toward the SGP. This strength is consistent with the strength of the GMF based on the observation or theoretical expectation [1][2][20]. If we assume the primary particle of a UHECR is a proton, the estimated strength of the GMF would be $17.60 \mu \mathrm{G}$, which is too strong to be considered to be a reasonable estimation. This result may support the idea that the primary particle of PAO would not be protons.

We examined the correlation between the arrival direction distribution of UHECRs observed by the PAO and the position of AGNs, as tracers of the LSS of the universe, toward the SGP. Introducing the comparison method of CADD and Kuiper statistic, we conducted a statistical comparison of the correlation through the Monte-Carlo simulations. It was found that the best-fit parameter for the smearing angle is $8^{\circ}$ if the source fraction is 0.7 . We interpret this angle as the deflection angle by the GMF toward the SGP. Considering the consistency with the outcome of the PAO's mass composition obtained by an analysis of the $X_{\max }$ distribution, the estimated strength of the GMF toward the SGP is about $2.5 \mu \mathrm{G}$ if we assumed the primary particles of UHECRs are nitrogen nuclei. Our results are consistent with the observations or theoretical expectations. We expect that we can open the era of UHECR astronomy with the UHECR data to be updated in the future.

\section{References}

[1] R. Beck, Galactic and extragalactic magnetic fields Space Science Reviews 99 (2001) 243 [astro-ph/0012402].

[2] R. Jansson and G.R. Farrar, The galactic magnetic field ApJL 761 (2012) 11 [arXiv: 1210 . 7820 ].

[3] D Ryu et al., Turbulence and magnetic fields in the large-scale structure of the universe Science $\mathbf{3 2 0}$ (2008) 909 [arXiv: 0805.2466$]$.

[4] K. Dolag et al., Constrained simulations of the magnetic field in the local Universe and the propagation of ultrahigh energy cosmic rays JCAP 01 (2005) 009 [ast ro-ph / 0410419 ].

[5] S. Brown, et al., Limiting magnetic fields in the cosmic web with diffuse radio emission MNRAS 468 (2017) 4246 [arXiv: 1703 . 07829]. 
[6] R.U. Abbasi et al., First observation of the Greisen-Zatsepin-Kuzmin suppression PRL 100 (2008) 101101 [astro-ph/0703099].

[7] J. Abraham et al., Observation of the suppression of the flux of cosmic rays above $4 \times 10^{19} \mathrm{eV} \mathrm{PRL}$ 101 (2008) 061101 [arXiv: 0806 .4302].

[8] T. Abu-Zayyad, et al., The cosmic-ray energy spectrum observed with the surface detector of the Telescope Array experiment ApJL 768 (2013) 1 [arXiv: 1205 . 5067].

[9] R.U. Abbasi et al., Indications of Proton-Dominated Cosmic-Ray Composition above 1.6 EeV PRL 104 (2010) 161101 [arXiv:0910.4184].

[10] A. Aab et al., Depth of maximum of air-shower profiles at the Pierre Auger Observatory. II. Composition implications PRD 90 (2014) 122006 [arXiv: 1409.5083 ].

[11] R.U. Abbasi, et al., Study of Ultra-High Energy Cosmic Ray composition using Telescope Array's Middle Drum detector and surface array in hybrid mode Astropart. Phys. 64 (2015) 49 [arXiv:1408.1726].

[12] T. Akahori et al., Simulated Faraday rotation measures toward high galactic latitudes ApJ 767 (2013) 150 [arXiv:1303.1595].

[13] M.P. Veron-Cetty and P. Veron, A catalogue of quasars and active nuclei: 13th edition A\&A 518 (2010) A10

[14] A. Aab et al., Searches for anisotropies in the arrival directions of the highest energy cosmic rays detected by the Pierre Auger Observatory ApJ 804 (2015) 15 [arXiv: 1411.6111].

[15] K. Greisen, End to the cosmic-ray spectrum? PRL, 16 (1966) 748

[16] G.T. Zatsepin and V.A. Kuz'min, Upper limit of the spectrum of cosmic rays JETPL 4 (1966) 78

[17] H.B. Kim and J. Kim, Statistical analysis of the correlation between active galactic nuclei and ultra-high energy cosmic rays JCAP 03 (2011) 006 [arXiv: 1009.2284 ].

[18] H.B. Kim and J. Kim, Revisit of correlation analysis between active galactic nuclei and ultra-high energy cosmic rays IJMPD 22 (2013) 1350045 [arXiv: 1203.0386 ].

[19] H.B.J. Koers and P. Tinyakov, Testing large-scale (an)isotropy of ultra-high energy cosmic rays JCAP 04 (2009) 003 [arXiv: 0812 . 0860].

[20] S.A. Mao et al., A survey of extragalactic faraday rotation at high galactic latitude: the vertical magnetic field of the milky way toward the galactic poles ApJ 714 (2010) 1170 [arXiv:1003.4519]. 